\section{Potential of butyrate to influence food intake in mice and men}

\author{
Kristina S Fluitman, ${ }^{1,2}$ Madelief Wijdeveld, ${ }^{3}$ Max Nieuwdorp, ${ }^{1,3}$ \\ Richard G IJzerman ${ }^{1}$
}

In recent years, many studies have been dedicated towards elucidating the role of the intestinal microbiota in metabolic health. It was suggested that specific intestinal microbial compositions can either protect from-or contribute to obesity and metabolic diseases. A causal relation between the microbiota and host metabolic profile was demonstrated when faecal microbiota of lean and obese subjects was transferred into mice, which then exhibited corresponding phenotypes. ${ }^{1}$ Short chain fatty acids (SCFAs) have broadly been proposed as key mediators in the microbiota-induced metabolic effects on the host. They are produced by bacterial fermentation of otherwise indigestible nutrients. The most abundant SCFAs are propionate, acetate and butyrate. All have been extensively studied in murine models for their possible influence on colonic health, glucose and lipid metabolism, as well as appetite and energy expenditure. Although various mechanisms by which these SCFAs exert their effect were suggested, ${ }^{2-7}$ the precise pathways remain unclear.

In Gut, Li et al report on the mechanisms by which butyrate positively influences energy balance and protects from diet-induced obesity. They deduce that many of the butyrate-induced metabolic benefits are driven by a decrease in energy intake. In their first experiment, Li et al exposed overnight fasted mice to either intravenously administered butyrate or to butyrate administered via intragastric gavage. Interestingly, only intragastric administration affected feeding behaviour, reducing cumulative 24 hours food intake by $21 \%$. Furthermore, it induced a marked shift in the activity of hypothalamic orexigenic and anorexic neurons. This demonstrates that butyrate administration

\footnotetext{
${ }^{1}$ Department of Internal Medicine, VU University Medical Center, Amsterdam, The Netherlands

${ }^{2}$ Wallenberg Laboratory, Department of Molecular and Clinical Medicine, Sahlgrenska Academy, University of Gothenburg, Göteborg, Sweden

${ }^{3}$ Department of Internal and Vascular Medicine, Academic Medical Center, Amsterdam, The Netherlands
}

Correspondence to Dr Richard G IJzerman, Department of Internal Medicine, VU University Medical Center, Amsterdam, 1081 HV, The Netherlands; rg. ijzerman@vumc.nl affects central appetite regulation before butyrate enters the systemic circulation. Next, Li et al conducted a 9-week trial, feeding mice a high fat diet (HFD) with or without $5 \%$ sodium butyrate. They again found a reduction in food intake of $22 \%$ in the butyrate-fed group, along with a reduction in body weight, gonadal white adipose tissue mass, liver weight and lipid content. A third group of mice was added, who received a HFD without butyrate, restricted to the amount of food that the butyrate-fed group ate. These pair-fed mice showed effects comparable to those in the butyrate group. However, not all effects were similarly present in the pair-fed group. Therefore, some, but not all effects of butyrate were thought to be mediated by a decrease in energy intake. In a final experiment, the effects of butyrate were abolished after subdiaphragmatic vagotomy surgery, suggesting that the gut-brain neural circuit is crucial.

The authors speculate that the orally administered butyrate reduces appetite by stimulating the secretion of glucagon-like peptide 1 (GLP-1) in the gut. GLP-1 would then stimulate the vagal nerve, which innervates the brainstem and induces satiety. However, endogenous GLP-1 concentrations were not measured and a previous study demonstrated a GLP-1-independent effect of highly fermentable supplements on appetite. ${ }^{2}$ To further explore the contribution of GLP-1 to these SCFA-mediated effects, GLP-1 levels could be measured or endogenous GLP-1 activity could be blocked by a GLP-1 receptor antagonist. Alternatively, the metabolic benefits of butyrate may be induced by satietogenic gut hormone Peptide $Y Y^{3}$ or by intestinal gluconeogenesis and increased portal glucose sensing by the vagal nerve.

Li et al conclude their report by proposing that butyrate, as oral supplement, is a promising strategy to combat obesity and related cardiometabolic diseases. However, we feel that this conclusion may be somewhat premature. First, validation in human studies is needed and may not yield as convincing results. Human physiology naturally differs from that of a mouse, and conditions surrounding a human dietary intervention are often not as standardised. In the last years, it was attempted to translate the promising, metabolically beneficial effects of antibiotics (as microbiota-manipulating strategy) from mice to humans. Results were far less pronounced. ${ }^{8}$ Second, as the authors rightly note in their article, the satietogenic effects of butyrate are not consistent across literature. Although Lin et $a l^{6}$ also found that butyrate reduced food intake, other studies did not, while still observing several metabolically beneficial effects. ${ }^{5}$ This suggests that a decrease in food intake does not mediate these metabolic benefits under all circumstances. It would be interesting to further explore which circumstances (like dosage, administration mode, endogenous SCFA production, baseline gut microbiota) are needed for butyrate to decrease food intake. Such knowledge would have important implications for any clinical application of butyrate as antiobesity drug. The authors suggest that the inconsistency in results may be caused by a difference in HFD type. Both studies that found a reduction in food intake used a similar lard-based HFD, ${ }^{69}$ while another study used a palm oil fat-based HFD, and did not find such a result. ${ }^{5} \mathrm{Li}$ et al briefly speculate that the different types of HFD might have differently impacted the intestinal microbiota, thereby the production of endogenous SCFAs, and the response to exogenous butyrate. They immediately dispute this hypothesis, however, as the chow-fed mice from the acute intervention also experienced an acute decrease in food intake. Nonetheless, we would like to argue that the mechanisms underlying the effects in the acute intervention might differ from those in the chronic dietary intervention. For instance, it could be speculated that the effects in the acute intervention were due to mechanical (bulk) effects of butyrate on the stomach. Butyrate could remain in the stomach longer than the vehicle that was infused in the control group. This would explain why the reduction in food intake was especially evident during the first hour after the intervention. Finally, one could wonder whether the results of individual mice were consistent across groups, or whether there were so-called responders and non-responders. If so, it would be interesting to explore the differences between responders and non-responders, further identifying the circumstances that are essential for an effect to occur. By doing so, a recently published study from our group was able to identify baseline 
microbial composition as an essential factor for the response to microbiota faecal transplantation. ${ }^{10}$

The current study clearly demonstrates that dietary butyrate is capable of decreasing food intake in mice. Furthermore, it elegantly shows that many of the metabolic benefits may be mediated by food restriction and that all effects require a functioning vagal nerve. However, inconsistency across literature concerning the satietogenic effect of butyrate precludes generalising these results to humans. We argue that well-controlled human studies should be performed examining under which circumstances and in which individuals microbiome-targeted or SCFA-targeted therapies are most effective. This will help optimise efficiency and select individuals that will benefit the most from such therapies.

Contributors MN, RGIJ and KSF were involved in the conceptualisation of the commentary. KSF and MW drafted the work. All authors have critically revised this commentary and approved the final version for publication.

Funding KSF is supported by the European Union Horizon 2020 PROMISS project 'PRevention Of Malnutrition In Senior Subjects in the EU' (grant agreement no. 678732). The content only reflects the author's view and the Commission is not responsible for any use that may be made of the information it contains; MW is supported by an AMC MD/ PhD Scholarship 2017-2018; MN is supported by a ZONMW-VIDI grant $2013(016.146 .327)$ and a Dutch Heart Foundation CVON Young Talent Grant 2013.

Competing interests $\mathrm{MN}$ is on the scientific advisory board of Caelus Health. No other competing interests declared.

Provenance and peer review Commissioned; internally peer reviewed.

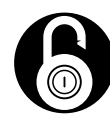

\section{OPEN ACCESS}

Open Access This is an Open Access article distributed in accordance with the Creative Commons Attribution Non Commercial (CC BY-NC 4.0) license, which permits others to distribute, remix, adapt, build upon this work non-commercially, and license their derivative works on different terms, provided the original work is properly cited and the use is noncommercial. See: http://creativecommons.org/licenses/ by-nc/4.0/

(C) Article author(s) (or their employer(s) unless otherwise stated in the text of the article) 2018. All rights reserved. No commercial use is permitted unless otherwise expressly granted.

\section{D) Check for updates}

To cite Fluitman KS, Wijdeveld M, Nieuwdorp M, et al. Gut 2018;67:1203-1204.

Received 8 December 2017

Revised 9 January 2018

Accepted 10 January 2018

Published Online First 30 January 2018

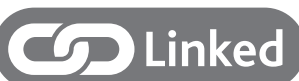

- http://dx.doi.org/10.1136/gutjnl-2017-314050

Gut 2018;67:1203-1204.

doi:10.1136/gutjnl-2017-315543

\section{REFERENCES}

1 Ridaura VK, Faith JJ, Rey FE, et al. Gut microbiota from twins discordant for obesity modulate metabolism in mice. Science 2013;341:1241214.

2 Anastasovska J, Arora T, Sanchez Canon GJ, et al. Fermentable carbohydrate alters hypothalamic neuronal activity and protects against the obesogenic environment. Obesity 2012;20:1016-23.

3 Brooks L, Viardot A, Tsakmaki A, et al. Fermentable carbohydrate stimulates FFAR2-dependent colonic PYY cell expansion to increase satiety. Mol Metab 2017:6:48-60.

4 Frost G, Sleeth ML, Sahuri-Arisoylu M, et al. The short-chain fatty acid acetate reduces appetite via a central homeostatic mechanism. Nat Commun 2014:5:3611.

5 den Besten G, Bleeker A, Gerding A, et al. ShortChain Fatty Acids Protect Against High-Fat Diet-Induced Obesity via a PPAR $\gamma$-Dependent Switch From Lipogenesis to Fat Oxidation. Diabetes 2015;64:2398-408.

6 Lin HV, Frassetto A, Kowalik EJ, et al. Butyrate and propionate protect against diet-induced obesity and regulate gut hormones via free fatty acid receptor 3 -independent mechanisms. PLoS One 2012;7:e35240

7 De Vadder F, Kovatcheva-Datchary P, Goncalves D, et al. Microbiota-generated metabolites promote metabolic benefits via gut-brain neural circuits. Cell 2014;156:84-96

8 Reijnders D, Goossens GH, Hermes GD, et al. Effects of Gut Microbiota Manipulation by Antibiotics on Host Metabolism in Obese Humans: A Randomized Double-Blind Placebo-Controlled Trial. Cell Metab 2016;24:63-74.

9 Li Z, Yi CX, Katiraei S, et al. Butyrate reduces appetite and activates brown adipose tissue via the gut-brain neural circuit. Gut 2018;67:1269-79.

10 Kootte RS, Levin E, Salojärvi J, et al. Improvement of Insulin Sensitivity after Lean Donor Feces in Metabolic Syndrome Is Driven by Baseline Intestinal Microbiota Composition. Cell Metab 2017;26:611-9. 